Original

\title{
Factores pronósticos en la supervivencia de los pacientes con carcinoma transicional de vejiga tratados con cistectomia radical
}

\author{
Juan I. Monzó Gardiner, Felipe Herranz Amo, José M. Díez Cordero, Ramiro Cabello Benavente, \\ Ángel Silmi Moyano*, Carlos Hernández Fernández
}

Hospital General Universitario Gregorio Marañón. *Hospital Clínico San Carlos. Madrid. España.

\begin{abstract}
Resumen
Objetivo: conocer las variables clínicas y patológicas que influyen en la mortalidad cáncer-específica de los pacientes con carcinoma transicional de vejiga tratados mediante cistectomía radical (CR).

Material y Método: análisis retrospectivo de 333 pacientes con cáncer transicional de vejiga tratados mediante CR. Se analizaron variables agrupadas en el período pre-cistectomía, peri-cistectomía y de seguimiento. Se definieron 4 grupos de riesgo en función del estadio patológico: a) Enfermedad localizada vesical sin afectación ganglionar (pTO-2, pNO); b) Enfermedad extravesical sin afectación ganglionar (pT3-4, pNO); c) Enfermedad con afectación ganglionar (pT0-4, pN+); d) Sin datos sobre la afectación ganglionar (pTO-4, pNx). Realizamos un análisis univariante y dos modelos de multivariante con y sin los grupos de riesgo descritos.

Resultados: La media de seguimiento de la serie fue de 52,6 $\pm 51(2-221)$ meses con una mediana de 31 meses. Un 7,2\% de los pacientes presentó estadio pT0, 12\% pT1, 26,7\% pT2, 34,5\% pT3 y un 19,5\% pT4. El 20,7\% de los pacientes tenían metástasis ganglionares (pN+). La supervivencia cáncer específica a los 5 años fue del $57 \%$ y del $54 \%$ a los 10 años.

Conclusiones: El estadio patológico, la afectación ganglionar y los grupos de riesgo se comportaron como factores predictivos independientes para la supervivencia cáncer-específica. La asociación por grupos de riesgo permite predecir de una forma más fiable el riesgo de fallecer por cáncer de vejiga e identificar a los pacientes en los que la cistectomía resulta un tratamiento insuficiente y que se podrían beneficiar de un tratamiento adyuvante.
\end{abstract}

Palabras claves: Cáncer de vejiga. Cistectomía radical. Supervivencia.

Prognostic factors for survival in patients with transitional baldder cancer treated with radical cystectomy

\section{Absctract}

Purpose: to recognize clinical and pathological variables that influence in bladder cancer specific mortality in patients with transitional bladder cancer treated with radical cystectomy.

Matherial and Method: retrospective analysis of 333 patients with transitional bladder cancer treated with radical cystectomy. Variables included during pre-cystectomy, peri-cystectomy and post-cystectomy period were analyzed.

Four groups were defined based on pathological state: a) Organ-confine bladder cancer without lymph node metastasis (pTO-2, pN0); b) Extravesical desease without lymph node metastasis (pT3-4, pNO); c) Bladder cancer with lymph node metastasis (pT0-4, pN+); d) No data of lymph node affection (pT0-4, pNx). Univariate analysis and two models of multivariate analysis were performed including the risk group as a variable in one the latest.

Results: Mean follow up was $52.6 \pm 51(2-221)$ months with a median of 31 months. Pathological state pTO was observed in $7.2 \%$ of the patients, $12 \%$ were pT1, $26.7 \%$ pT2, $34.5 \%$ pT3 and $10.5 \%$ pT 4 . Lymph node metastasis was detected in $20.7 \%$ of the patients. Lymph node metastasis increased according to pathological state rises.

Five and 10 years specific survival was $57 \%$ and $54 \%$ respectively.

Conclusions: Local pathological state, lymph node status and risk groups were independent predictive factors for bladder cancer specific survival. Risk group association is a reliable method to predict bladder cancer specific survival and to identify the suitable patient group to get benefit from adjuvant therapy.

Keywords: Bladder cancer. Radical cystectomy. Survival.

$\mathrm{E}$ 1 cáncer de vejiga es el séptimo tumor más frecuente en el mundo, representando el 3,2\% del total de todos los tumores. La incidencia más alta para ambos sexos se observa en Europa, Norteamérica y Australia ${ }^{1,2}$.
Es el cuarto tumor en incidencia en el varón (7\% del total de los tumores) y la octava causa de muerte oncológica (3\% del total de los tumores). En la mujer no figura entre los tumores más frecuentes $^{3}$. 
El tipo histológico más frecuente de cáncer de vejiga es el carcinoma transicional, derivado del urotelio, que constituye más del 90\% de los casos en Europa, Norteamérica y Australia ${ }^{4}$.

De los 63.210 nuevos cánceres de vejiga diagnosticados en 2005, un tercio de los mismos invaden la muscular propia y otro $15-30 \%$ de los tumores superficiales de alto grado progresarán a infiltrantes, la mayoría dentro de los 5 años siguientes al diagnóstico. La cistectomía radical con linfadenectomía pélvica es el tratamiento estándar para el cáncer vesical infiltrante ${ }^{5}$.

A pesar de la mejoría en las técnicas y cuidados postoperatorios, el análisis de las series anteriores y posteriores a 1986, año en el que se introduce el cisplatino en los esquemas de tratamiento con quimioterapia, revelan sólo una modesta mejoría en la supervivencia ${ }^{6}$.

Aproximadamente el $50 \%$ de los pacientes con cáncer urotelial infiltrante de vejiga fallecen en los 3-4 años siguientes a la cistectomía radical. Se cree que esto es debido a que en el momento de la cistectomía la mitad de los pacientes presentan metástasis a distancia no sospechadas (micrometástasis). La cistectomía se considera como un tratamiento insuficiente para la curación de los pacientes con enfermedad extravesical sin o con afectación ganglionar (pT3-4, pNO/pN+) $)^{5,7-13}$. Los factores pronósticos para la supervivencia descritos incluyen el tamaño tumoral, márgenes quirúrgicos, la clasificación TNM, número de ganglios afectados y la invasión linfovascular ${ }^{5,13-15}$. Pero destacan entre todos estos el estadio patológico local y la afectación ganglionar.

El mayor conocimiento de los factores de riesgo de la población española intervenida en hospitales públicos así como de la utilidad de la quimioterapia adyuvante en estos pacientes, resulta de gran interés para ser comparados con la series publicadas hasta el momento.

\section{OBJETIVO}

Conocer cuales son las variables clínicas y patológicas que influyen en la mortalidad cáncer-específica de los pacientes con carcinoma transicional de vejiga tratados mediante cistectomía radical.

\section{MATERIAL Y MÉTODO}

Análisis retrospectivo de 397 pacientes con cáncer transicional de vejiga tratados mediante cistec- tomía radical en el periodo de tiempo comprendido entre 1986 y 2005. Se excluyeron 64 (16\%) pacientes por las siguientes razones: $21(5,3 \%)$ por exitus en el postoperatorio inmediato, $32(8 \%)$ por radioterapia radical previa con intención curativa, $6(1,5 \%)$ por quimioterapia neoadyuvante y $5(1,2 \%)$ pacientes procedentes de otras Comunidades Autónomas que realizaron el seguimiento en su Centro de referencia. Por lo tanto, quedaron 333 pacientes válidos para el estudio.

La revisión de las historias clínicas se realizó entre octubre del 2006 y julio del 2007.

Las indicaciones para la cistectomía no han cambiado durante el periodo de tiempo del estudio. Se indicó la cistectomía en los pacientes con carcinoma vesical infiltrante (cT2 o superior), tumor superficial (cT1 o menor) incontrolable endoscópicamente, carcinoma "in situ" (Tis) resistente a BCG y persistencia o recidiva de tumor vesical después de radioterapia radical.

Se realizó en ambos sexos una linfadenectomía pélvica estándar junto con la cistectomía radical. No obtuvimos el número de ganglios extraídos ya que este dato estaba ausente en algunos de los informes anatomopatológicos.

Todos los pacientes habían sido sometidos al menos a una resección transuretral previa confirmando la existencia de un carcinoma transicional primario de vejiga así como su grado histológico. A partir del 2002 se clasificaron patológicamente según la clasificación TNM 2002 (6 $6^{\mathrm{a}}$ ed.) de la UICC. Los pacientes tratados con anterioridad al 2002 fueron reclasificados según la clasificación del 2002. Los pacientes sin tumor en la pieza de cistectomía fueron clasificados como pTO. Cuando existió invasión de los linfáticos se agruparon como $\mathrm{pN}+$. Los pacientes en los que no se realizó linfadenectomía se clasificaron como pNx.

Se analizaron las siguientes variables:

- Pre-cistectomía: edad, sexo, estadio clínico (cTNM), comorbilidad por aparatos, estado físico según la American Society of Anesthesiologits (ASA), hidronefrosis y necesidad de nefrostomía y fecha de la resección transuretral (RTU).

- Peri-cistectomía: fecha de la cistectomía, cirujano (MIR/Staff), tipo de derivación urinaria, estadio patológico (pTNM).

- Seguimiento: administración de quimioterapia adyuvante, situación del paciente en el momento de la revisión, fecha última revisión y causa del exitus. 
Se definieron 4 grupos de riesgo en función de los hallazgos patológicos en la vejiga y en los ganglios de la linfadenectomía: a) Enfermedad localizada vesical sin afectación ganglionar (pTO-2, pNO); b) Enfermedad extravesical sin afectación ganglionar (pT3-4, pNO); c) Enfermedad con afectación ganglionar (pT0-4, pN+); d) No existen datos sobre la afectación ganglionar (pTO-4, pNx).

Los pacientes con pTa (2 casos) y con pTis (4 casos) se incluyeron en la clasificación como pT1.

Los criterios para la administración de quimioterapia adyuvante han ido modificándose durante el periodo del estudio. Al principio se administró a los pacientes al menos con infiltración de la muscular profunda (pT2b) o afectación ganglionar ( $\mathrm{pN}+$ ), actualmente sólo se administra a los pacientes con estadio pT3 o superior o pN+. Se administraron 3 ciclos de MVAC (metotrexate, vinblastina, adriamicina y cisplatino), posteriormente se sustituyó el cisplatino por carboplatino.

Los pacientes fueron seguidos en la consulta externa a los 3 meses y cada 6 meses hasta el $5^{\circ}$ año. Posteriormente se les realizó un seguimiento anual.

El test exacto de Fisher y la prueba de la Chi cuadrado se utilizaron para evaluar la asociación entre variables categóricas. Para evaluar las diferencias entre las variables con una distribución continua, se utilizó la $\mathrm{t}$ de Student o el test de Mann-Whitney.

El método de Kaplan-Meier se ha utilizado para evaluar la supervivencia y el test de long rank para evaluar sus diferencias entre los distintos grupos con sus intervalos de confianza al 95\%.

Se realizó un análisis univariante y posteriormente se ajustó mediante un modelo de riesgos proporcionales de Cox (método ENTER).

La significación estadística se consideró cuando existió una $\mathrm{p}<0,05$. Todos los cálculos se realizaron con el programa estadístico SPSS versión 12.0 en castellano.

\section{RESULTADOS}

De los 333 pacientes válidos para el estudio, el $90,7 \%$ fueron hombres y el 9,3\% mujeres. La edad media fue de 63,4 \pm 9 (31 - 89) con una mediana de 65 años. La cistectomía fue realizada por un Staff en $212(63,6 \%)$ pacientes y en los 121 (36,3\%) restantes por un MIR ayudado por un Staff. Las características clínicas se resumen en la Tabla 1 y las características patológicas en la Tabla 2.
Tabla 1. Características clínicas

\begin{tabular}{lc}
\hline Variables & $\mathbf{n}(\%)$ \\
\hline Sexo: & $302(90,7)$ \\
$\quad$ Hombre & $31(9,3)$ \\
Mujer & \\
Grupos de edad (años): & $104(31,2)$ \\
$\quad$ <0 & $155(46,5)$ \\
$61-70$ & $74(22,2)$ \\
> 70 & \\
Estado físico (ASA): & $65(19,5)$ \\
I & $191(57,4)$ \\
II & $37(11,1)$ \\
III & $40(12)$ \\
Sin clasificar & \\
Estadio clínico: & $3(0,9)$ \\
cTx & $2(0,6)$ \\
cTis & $31(9,3)$ \\
cT1 & $277(83,2)$ \\
cT2 & $7(2,1)$ \\
cT3 & $13(3,9)$ \\
cT4 & \\
Hidronefrosis: & $95(28,5)$ \\
Si & $63(66,3)$ \\
Unilateral & $32(33,7)$ \\
Bilateral &
\end{tabular}

Tabla 2. Características patológicas

\begin{tabular}{|c|c|}
\hline Variables & n (\%) \\
\hline \multicolumn{2}{|l|}{ Estadio Patológico: } \\
\hline pTO & $24(7,2)$ \\
\hline pT1 & $40(12)$ \\
\hline pT2 & $89(26,7)$ \\
\hline pT3 & $115(34,5)$ \\
\hline pT4 & $65(19,5)$ \\
\hline \multicolumn{2}{|l|}{ Grado citológico: } \\
\hline G2 & $22(6,6)$ \\
\hline G3 & $311(93,4)$ \\
\hline \multicolumn{2}{|l|}{ Afectación Linfática: } \\
\hline $\mathrm{pNx}$ & $19(5,7)$ \\
\hline pNO & $245(73,6)$ \\
\hline $\mathrm{pN}+$ & $69(20,7)$ \\
\hline \multicolumn{2}{|l|}{ Grupos de Riesgo: } \\
\hline pTO-2, pNO & $141(42,3)$ \\
\hline pT3-4, pNO & $104(31,2)$ \\
\hline pTO-4, pN+ & $69(20,7)$ \\
\hline pT0-4, pNx & $19(5,7)$ \\
\hline \multicolumn{2}{|l|}{ Derivación urinaria: } \\
\hline Ureterostomía cutánea & $31(9,3)$ \\
\hline Conducto ileal (Bricker) & $236(70,9)$ \\
\hline Continente externa & $2(0,6)$ \\
\hline Ureterosigmoidostomía tipo Mainz II & $18(5,4)$ \\
\hline Sustitución vesical ortotópica & $46(13,8)$ \\
\hline \multicolumn{2}{|l|}{ Cirugía asociada: } \\
\hline $\mathrm{Si}$ & $37(11,1)$ \\
\hline No & $296(88,9)$ \\
\hline \multicolumn{2}{|l|}{ Tipo de cirugía asociada: } \\
\hline Uretrectomía & $23(62,2)$ \\
\hline Nefroureterectomía & $9(24,3)$ \\
\hline Prótesis aorto-ilíaca & $2(5,4)$ \\
\hline Apendicectomía & $2(5,4)$ \\
\hline Hernioplastia & $1(2,7)$ \\
\hline \multicolumn{2}{|l|}{ Quimioterapia adyuvante: } \\
\hline $\mathrm{Si}$ & $134(40,2)$ \\
\hline No & $199(59,8)$ \\
\hline
\end{tabular}


La media de seguimiento de la serie fue de 52,6 \pm 51 (2-221) meses con una mediana de 31 meses.

Durante el seguimiento 135 (40,5\%) pacientes presentaron complicaciones relacionadas con la cistectomía. La más frecuente fue la estenosis de la anastomosis uretero-ileal en 48 (14,4\%) pacientes, hernia incisional en 23 (6,9\%) y obstrucción-suboclusión intestinal en otros $23(6,9 \%)$ casos. En 71 $(21,3 \%)$ pacientes fue necesaria la cirugía para el tratamiento de las complicaciones.

En $24(7,2 \%)$ pacientes no se encontró tumor en la pieza de cistectomía (pT0). El 20,7\% de los pacientes tenían metástasis ganglionares $(\mathrm{pN}+)$. La afectación ganglionar $(\mathrm{pN}+)$ aumentó según se incrementó el estadio patológico local (pT) (Tabla 3). El 37,8\% de los pacientes con enfermedad extravesical tenían afectación ganglionar, mientras que sólo el 4,8\% de los pacientes con enfermedad órganoconfinada eran $\mathrm{pN}(+)$.

Tabla 3. Relación entre Estadio Local (pT) y la Afectación Ganglionar (pN)

\begin{tabular}{lccc}
\hline & pN0 n (\%) & pN+ n (\%) & pNx n (\%) \\
\hline pT0 & $24(100)$ & 0 & 0 \\
pT1 & $32(86,5)$ & $3(8,1)$ & $2(5,4)$ \\
pT2 & $83(93,3)$ & $4(4,5)$ & $2(2,2)$ \\
pT3 & $72(63,2)$ & $35(30,7)$ & $7(6,1)$ \\
pT4 & $30(46,2)$ & $27(41,5)$ & $8(12,3)$ \\
Total & $245(73,6)$ & $69(20,7)$ & $19(5,7)$ \\
\hline
\end{tabular}

Al 40,2\% de los pacientes se les administró quimioterapia adyuvante. El 28\% de los pacientes con enfermedad localizada fueron tratados con quimioterapia adyuvante, el 57,5\% de los pacientes con enfermedad extravesical y el $82,6 \%$ de los pacientes con afectación ganglionar en la linfadenectomía.

Al final de la revisión 143 (43\%) pacientes se encontraban en remisión completa (libre de enfermedad), 21 (6\%) presentaban recidiva local y/o a distancia y 169 (51\%) habían fallecido. De los pacientes fallecidos, 130 (77\%) lo hicieron a consecuencia del cáncer de vejiga, $12(7 \%)$ por otro tumor y 27 (16\%) por otras causas.

La supervivencia media global de la serie fue de 103 (IC95\%, 91-115) meses, con una mediana de 61 (IC95\%, 27-95) meses. A los 5 años la supervivencia fue del 50\% y del 43\% a los 10 años (Fig. 1).

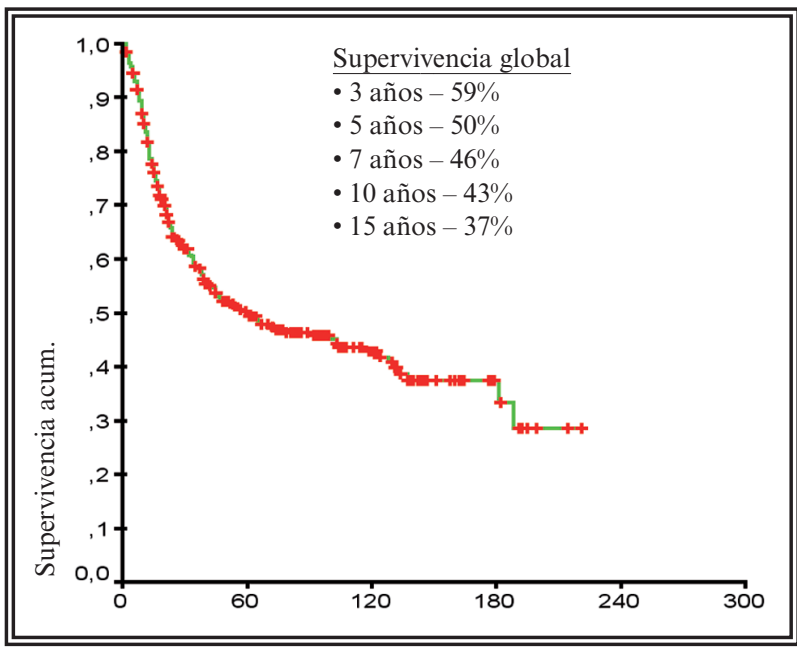

FIGURA 1. Supervivencia global.

La supervivencia media en los pacientes fallecidos según la causa fue de: 21 (IC95\%, 18-24) meses, con una mediana de 15 (IC95\%, 13-17) meses por cáncer de vejiga; 69 (IC95\%, 33-105) meses, con una mediana de 45 (IC95\%, 30-60) meses por otro tumor y 48 (IC95\%, 30-66), con una mediana de 24 (IC95\%, 30-66) meses cuando fallecieron por otras causas.

No se observaron diferencias significativas $(\mathrm{p}=0,44)$ en la supervivencia entre los diferentes estadios que se agrupan en la enfermedad organoconfinada (pTispT2), ni entre los grupos (pT3-pT4) que constituyen la enfermedad extravesical $(\mathrm{p}=0,11)$.

La supervivencia media cáncer específica de la serie fue de 129 (IC95\%, 117-141) meses. A los 5 años la supervivencia fue del 57\% y del 54\% a los 10 años (Fig. 2).

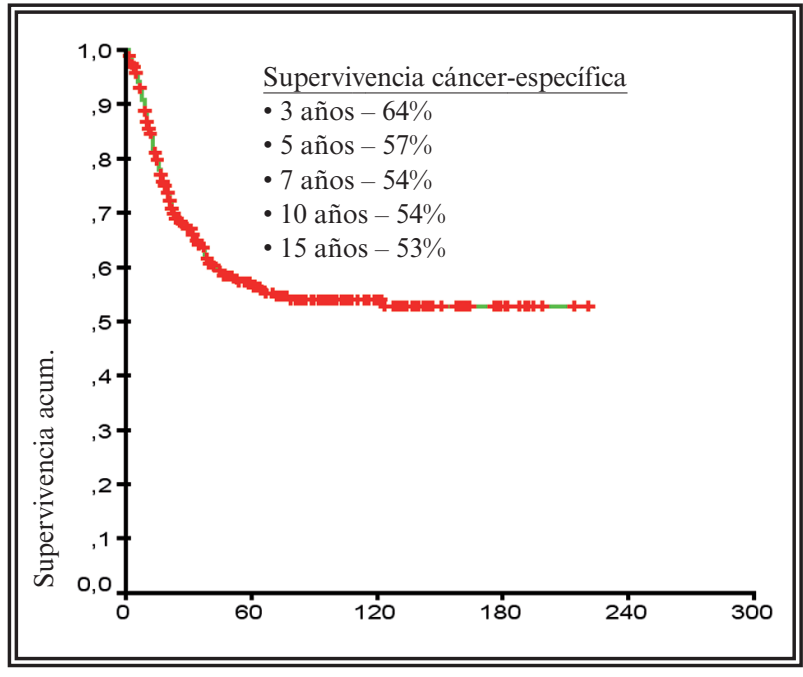

FIGURA 2. Supervivencia cáncer-específica. 
En el análisis univariante (regresión de Cox) la hidronefrosis, el grado citológico en la pieza de cistectomía, el estadio local patológico (pT), la afectación linfática $(\mathrm{pN}+)$, el grupo de riesgo y la administración de quimioterapia adyuvante se asociaron de forma significativa con una menor supervivencia cáncer-específica. Existiendo una tendencia importante a la significación en la edad $(\mathrm{p}=0,07)$ y en la clasificación según el estado físico (ASA) ( $\mathrm{p}=0,07)$ (Tabla 4).

En el análisis multivariante se introdujeron las variables significativas detectadas en el análisis univariante (la hidronefrosis, el grado citológico en la pieza de cistectomía, el estadio patológico local (pT), la afectación linfática $(\mathrm{pN}+)$ y la administración de quimioterapia adyuvante), así como la edad y el estado físico ya que presentaban una importante tendencia a la significación ( $\mathrm{p}=0,07)$ (Tabla 5).

$\mathrm{El}$ análisis multivariante identificó como variables independientes significativamente asociadas con la mortalidad cáncerespecífica al estadio patológico local (pT) y a la afectación ganglionar (pN). Se observó una tendencia en el mismo sentido con el aumento en el grado patológico $(p=0,07)$. La administración de quimioterapia presentó una tendencia $(p=0,08)$ a la disminución de la mortalidad cáncer-específica.

Se construyó un segundo modelo agrupando el estadio local patológico (pT) y la afectación linfática (pN) en Grupos de Riesgo (Tabla 6).

En este segundo modelo se observó que sólo el Grupo de Riesgo ( $<<0,001)$ se comportó como variable predictora independiente asociadas a la mortalidad cáncer-específica. La edad superior a 70 años ( $p=0,17)$, el grado citológico G3 en la pieza de cistectomía $(p=0,06)$ presentaron una tendencia al aumento de la mortalidad cáncer-específica. La
Tabla 4. Análisis univariante de las variables clínicas y patológicas que predicen la mortalidad cáncer-específica

\begin{tabular}{|c|c|c|c|}
\hline Variable & Riesgo Relativo & IC $95 \%$ & Valor de $p$ \\
\hline Edad: & & & 0,07 \\
\hline$<60$ años & 1,000 & Referencia & \\
\hline $61-70$ años & 1,164 & $0,778-1,744$ & 0,46 \\
\hline > 70 años & 1,730 & $1,067-2803$ & 0,02 \\
\hline \multicolumn{4}{|l|}{ Sexo: } \\
\hline Varón & 1,000 & Referencia & \\
\hline Mujer & 1,404 & $0,820-2,406$ & 0,21 \\
\hline Estado físico (ASA): & & & 0,07 \\
\hline ASA I & 1,000 & Referencia & \\
\hline ASA II & 1,715 & $1,036-2,840$ & 0,03 \\
\hline ASA III & 1,996 & $0,007-3,998$ & 0,05 \\
\hline \multicolumn{4}{|l|}{ Hidronefrosis: } \\
\hline No & 1,000 & Referencia & \\
\hline $\mathrm{Si}$ & 1,665 & $1,162-2,387$ & 0,006 \\
\hline Estadio Clínico (cT): & & & 0,91 \\
\hline cT1 & 1,000 & Referencia & \\
\hline cT2 & 1,062 & $0,556-2,032$ & 0,85 \\
\hline cT3 & 1,054 & $0,230-4,830$ & 0,94 \\
\hline cT4 & 1,373 & $0,539-3,493$ & 0,50 \\
\hline Tiempo entre RTU-Cistectomía & 1,049 & $0,941-1,170$ & 0,39 \\
\hline Grado patológico: & & & 0,006 \\
\hline GO & 1,000 & Referencia & \\
\hline G2 & 0,671 & $0,123-3,662$ & 0,64 \\
\hline G3 & 3,324 & $1,228-8,999$ & 0,01 \\
\hline Estadio patológico (pT): & & & $<0,001$ \\
\hline pT1 & 0,856 & $0,241-3,034$ & 0,81 \\
\hline pT2 & 1,806 & $0,628-5,189$ & 0,27 \\
\hline pT3 & 4,045 & $1,416-11,160$ & 0,007 \\
\hline pT4 & 7,603 & $2,692-21,257$ & $<0,001$ \\
\hline Afectación linfática (pN): & & & $<0,001$ \\
\hline pNO & 1,000 & Referencia & \\
\hline $\mathrm{pN}+$ & 2,810 & $1,916-4,122$ & $<0,001$ \\
\hline $\mathrm{pNx}$ & 3,391 & $1,841-6,246$ & $<0,001$ \\
\hline Grupo de Riesgo: & & & $<0,001$ \\
\hline pTO-2, pNO & 1,000 & Referencia & \\
\hline pT3-4, pNO & 2,576 & $1,635-4,059$ & $<0,001$ \\
\hline pTO-4, pN+ & 4,413 & $2,765-7,043$ & $<0,001$ \\
\hline pTO-4, pNx & 5,326 & $2,733-10,379$ & $<0,001$ \\
\hline \multicolumn{4}{|l|}{ QT Adyuvante: } \\
\hline No & 1,000 & Referencia & \\
\hline $\mathrm{Si}$ & 1,452 & $1,028-2,057$ & 0,03 \\
\hline
\end{tabular}

administración de quimioterapia mantuvo la misma tendencia $(\mathrm{p}=0,08)$ en la disminución de la mortalidad cáncer-específica.

\section{DISCUSIÓN}

El cáncer de vejiga es una enfermedad con mayor incidencia en personas de más de 50 años de edad, siendo su máxima presentación entre la sexta y séptima década de la vida. En este trabajo observamos que la edad media es de 63,4 años y resulta muy similar a las edades de la mayoría de las diferentes series publicadas hasta la fecha ${ }^{5,9,12-19}$. 
Tabla 5. Análisis multivariante de las variables clínicas y patológicas que predicen la existente en nuestro entorno a mortalidad cáncer-específica

\begin{tabular}{|c|c|c|c|}
\hline Variable & Riesgo Relativo & IC 95\% & Valor de $p$ \\
\hline Edad: & & & 0,62 \\
\hline$<60$ años & 1,000 & Referencia & \\
\hline $61-70$ años & 1,245 & $0,778-1,990$ & 0,36 \\
\hline$>70$ años & 1,260 & $0,710-2,235$ & 0,43 \\
\hline Estado físico (ASA): & & & 0,80 \\
\hline ASA I & 1,000 & Referencia & \\
\hline ASA II & 1,197 & $0,702-2,039$ & 0,50 \\
\hline ASA III & 1,154 & $0,547-2,432$ & 0,70 \\
\hline \multicolumn{4}{|l|}{ Hidronefrosis: } \\
\hline No & 1,000 & Referencia & \\
\hline $\mathrm{Si}$ & 1,047 & $0,685-1,601$ & 0,83 \\
\hline \multicolumn{4}{|l|}{ Grado patológico: } \\
\hline $\mathrm{G} 2$ & 1,000 & Referencia & \\
\hline G3 & 3,602 & $0,866-14,988$ & 0,07 \\
\hline Estadio Patológico (pT): & & & $<0,001$ \\
\hline pTO & 1,000 & Referencia & \\
\hline pT1 & 0,632 & $0,167-2,393$ & 0,49 \\
\hline pT2 & 1,404 & $0,469-4,209$ & 0,54 \\
\hline pT3 & 2,755 & $0,936-8,110$ & 0,06 \\
\hline pT4 & 4,665 & $1,496-14,542$ & 0,008 \\
\hline Afectación ganglionar: & & & 0,005 \\
\hline NO & 1,000 & Referencia & \\
\hline $\mathrm{N}+$ & 2,091 & $1,212-3,608$ & 0,008 \\
\hline $\mathrm{Nx}$ & 2,388 & $1,2344-4,623$ & 0,01 \\
\hline \multicolumn{4}{|l|}{ QT Adyuvante: } \\
\hline No & 1,000 & Referencia & \\
\hline $\mathrm{Si}$ & 0,651 & $0,399-1,061$ & 0,08 \\
\hline
\end{tabular}
la hora de indicar la cistectomía en aquellos pacientes con tumor no infiltrante, manejando a los mismos mediante resecciones transuretrales iterativas y quimioterapia/inmunoterapia intravesical durante más tiempo.

En nuestra serie la proporción de pacientes con estadio pT4 $(19,5 \%)$ es superior a la media de la literatura. Esto puede ser debido a que en nuestro servicio, en los pacientes localmente avanzados, indicamos la cistectomía como tratamiento paliativo. Pero no podemos descartar que en España los pacientes acudan al Urólogo más tardíamente, ya que en la literatura española encontramos una serie de cistectomía radical del Complejo Hospitalario Universitario del Hospital de Albacete $^{19}$, quienes tienen un $31 \%$ de pacientes con estadio Tabla 6. Análisis multivariante de las variables clínicas y patológicas que predicen la mortalidad cáncer-específica utilizando la variable grupo de riesgo

\begin{tabular}{|c|c|c|c|}
\hline Variable & Riesgo Relativo & IC $95 \%$ & Valor de $\mathbf{p}$ \\
\hline Edad: & & & 0,36 \\
\hline$<60$ años & 1,000 & Referencia & \\
\hline $61-70$ años & 1,307 & $0,822-2,076$ & 0,25 \\
\hline > 70 años & 1,464 & $0,842-2,545$ & 0,17 \\
\hline Estado físico (ASA): & & & 0,31 \\
\hline ASA I & 1,000 & Referencia & \\
\hline ASA II & 1,479 & $0,886-2,469$ & 0,13 \\
\hline ASA III & 1,517 & $0,734-3,134$ & 0,26 \\
\hline Hidronefrosis: & & & 0,35 \\
\hline No & 1,000 & Referencia & \\
\hline $\mathrm{Si}$ & 1,220 & $0,933-15,924$ & \\
\hline Grupo de Riesgo: & & & $<0,001$ \\
\hline pTO-2, pNO & 1,000 & Referencia & \\
\hline pT3-4,pNO & 2,351 & $1,365-4,049$ & 0,002 \\
\hline pT0-4,pN+ & 4,700 & $2,454-9,003$ & $<0,001$ \\
\hline pT0-4, pNx & 4,570 & $2,213-9,441$ & $<0,001$ \\
\hline \multicolumn{4}{|l|}{ QT Adyuvante: } \\
\hline No & 1,000 & Referencia & \\
\hline $\mathrm{Si}$ & 0,651 & $0,398-1,065$ & 0,08 \\
\hline
\end{tabular}
$\leq$ pT2, 37,9\% de pT3 y $31,1 \%$ de pT4. Esta alta proporción de enfermedad pT4 podría explicarse por cualquiera de los argumentos anteriormente esgrimidos.

El 20,7\% de nuestros pacientes tenían metástasis ganglionares en el momento de la cirugía y dicha incidencia es similar a las publicadas en otros trabajos donde la afectación ganglionar se encuentra entre el $13 \%$ y el $33,1 \% 5,9,12-14,16,1719-23$.

A pesar de las mejoras en los cuidados perioperatorios, técnicas quirúrgicas, protocolos de quimioterapia adyuvante más

En nuestra serie tenemos un menor porcentaje de pacientes con estadio pT1 (12\%) con respecto diferentes series publicadas ${ }^{5,12-18,20}$, cuyos valores oscilan alrededor del $20 \%$. Tal vez, la explicación para dicha diferencia, esté en la menor agresividad efectivos y con menos efectos secundarios, el aumento de la supervivencia cáncer-específica ha sido pequeño ${ }^{6}$. Nuestros resultados reflejan la contundencia de esta enfermedad al tener una supervivencia global a los 5 años del $50 \%$ y del $43 \%$ a los 10 
años. A los 3 años sólo el 59\% de los pacientes estaban vivos, de lo que se infiere que el período de mayor riesgo de muerte para los pacientes sometidos a cirugía está dentro de los primeros 5 años y con especial énfasis en los primeros 3 años. Una vez superado este límite de tiempo, la probabilidad de supervivencia es mayor. En numerosas series donde se expresa este parámetro, la supervivencia es prácticamente idéntica entre uno y otro autor y están entorno a los valores de nuestra población en estudio $^{5,7-13}$.

En nuestra serie la supervivencia cáncer-específica a los 3, 5 y 10 años resultó ser del 64\%, 57\% y $54 \%$ respectivamente y similar a la de otros autores cuyos valores se encuentran entre el $44 \%$ y el $72 \%$ a los 5 años $8,11,13,14,20$.

En esta revisión los pacientes que fallecieron durante el seguimiento por cáncer de vejiga, el 83\% lo hicieron en los tres primeros años y el 95\% en los 5 años post-cistectomía. Soloway et $\mathrm{al}^{7}$ obtuvieron una mortalidad del $20 \%$ a los 2 años. Para Nishiyama et $\mathrm{al}^{9}$ el $79 \%$ del los pacientes fallecidos antes de los 5 años lo hicieron dentro de los 3 primeros. En la serie de Shariat et $\mathrm{al}^{14}$ la supervivencia cáncer-específica a los 3 años fue del 63,3\%. En otra serie de 124 pacientes se observó una supervivencia libre de recurrencia a los tres años del 77,1\% y del $75,6 \%$ a los 5 años ${ }^{22}$.

Para Segura Martín et al la probabilidad de supervivencia global a los 3 años es del $41,71 \%$ y a los 5 años es del $35,20 \%{ }^{19}$.
En la Tabla 7 comparamos las variables independientes en la supervivencia del cáncer de vejiga tratados con cistectomía expresadas en la literatura. El estadio patológico y la afectación ganglionar resultaron ser los factores de riesgo independientes para mortalidad cáncer específica y estas variables se repiten en otras series con resultados similares a los obtenidos en nuestro trabajo. En un trabajo español se analizaron retrospectivamente 298 pacientes tratados con cistectomía para establecer grupos de riesgo Se utilizaron las variables: afectación ganglionar, estadio patológico y afectación del estroma prostático, que resultaron ser predictoras de mortalidad por cáncer en el análisis univariante ${ }^{24}$.

En nuestro trabajo, la quimioterapia adyuvante se asocia a peor supervivencia en el análisis univariante debido a que los pacientes con peor pronóstico son los que reciben este tratamiento. En el análisis multivariante tiene una tendencia a disminuir la mortalidad cáncer específica. Existe un elevado porcentaje de pacientes (17\%) con enfermedad organoconfinada que recibieron tratamiento adyuvante con quimioterapia y podría tratarse de un sobretratamiento teniendo en cuenta que sólo parecen beneficiarse aquellos pacientes con afectación extravesical (estadio pT3-pT4 y/o N+2 25-28. Estos últimos cuatro estudios prospectivos citados son los que han orientado a los urólogos y oncólogos al uso de la quimioterapia adyuvante, pero son seriamente cuestionados por sus numerosos errores metodológicos ${ }^{29}$. Las guías para en manejo del cáncer de vejiga infiltrante de la

Tabla 7. Variables independientes en la supervivencia del cáncer de vejiga tratados con cistectomía. Revisión de la literatura

\begin{tabular}{|c|c|c|c|c|c|c|}
\hline & Estadio pT & $\begin{array}{l}\text { Afectación } \\
\text { ganglionar }\end{array}$ & Hidronefrosis & Edad & $\begin{array}{c}\text { Grado } \\
\text { citológico }\end{array}$ & Sexo \\
\hline Hautmann ${ }^{15}$ & si & si & si & no & no & - \\
\hline Hara $^{16}$ & si & si & - & no & no & no \\
\hline Harada $^{21}$ & si & si & - & si & no & no \\
\hline Louis-Johnsun 22 & si & si & no & no & no & no \\
\hline Bassi $^{18}$ & si & si & - & - & no & no \\
\hline Millar 23 & si & si & - & & si & no \\
\hline Dalbagni $^{20}$ & si & no & - & no & - & no \\
\hline Nishiyama $^{9}$ & si & si & - & si & no & no \\
\hline Cheng ${ }^{13}$ & si & si & - & no & no & no \\
\hline Segura-Martin 19 & si & si & si & no & no & no \\
\hline Antune $z^{17}$ & si & si & - & no & no & no \\
\hline Frazier $^{8}$ & si & si & - & si & no & no \\
\hline Shariat $^{14}$ & si & si & - & - & no & \\
\hline
\end{tabular}


$\mathrm{AEU}^{30}$ determinan que aun falta evidencia científica que demuestre el beneficio de la quimioterapia adyuvante en esta enfermedad. Trabajos como el nuestro descubren la necesidad de realizar estudios prospectivos y aleatorizados que den respuesta la utilidad de este tratamiento.

Este estudio adolece de las siguientes limitaciones: a) es una serie retrospectiva; b) comprende un largo período de estudio de 20 años; c) la variabilidad entre los cirujanos y el número considerable de cistectomías realizadas por los médicos internos residentes; d) durante el período de estudio las indicaciones de la quimioterapia han ido variando.

\section{CONCLUSIONES}

1. La supervivencia global y cáncer-específica, con una mediana de seguimiento de 61 meses, a los 5 años y 10 años fue del 50\% y 57\% y del 43\% y $54 \%$ respectivamente.

2. En el primer modelo de riesgos proporcionales de Cox (análisis multivariante), sólo el estadio patológico $(p<0,001)$ y la afectación ganglionar $(p=0,005)$ se comportaron como factores predictivos independientes en relación con la supervivencia cáncer-específica.

3. Para la construcción del segundo modelo de Cox, se han agrupo las variables pT y pN según la práctica clínica habitual (grupos de riesgo pT,pN). En este modelo observamos que según descendemos de un grupo de riesgo al siguiente, el riesgo relativo de fallecer por cáncer de vejiga se duplica (pTO-2, pNO, $\mathrm{RR}=1$; pT3-4, pN0, RR=2,351, p=0,002; pTO-4, pN+, $\mathrm{RR}=4,570, \mathrm{p}<0,001)$.

4. En ambos modelos el grado patológico y la quimioterapia adyuvante sólo muestran una tendencia a la significación estadística en relación con la supervivencia cáncer-específica. La tendencia de la quimioterapia adyuvante se puede definir como "protectora" ya que disminuye la mortalidad cáncerespecífica $(R R=0,651, p=0,08)$.

5. La asociación por grupos de riesgo permite predecir de una forma más fiable el riesgo de fallecer por cáncer de vejiga e identificar a los pacientes en los que la cistectomía resulta un tratamiento insuficiente y que se podrían beneficiar de un tratamiento adyuvante.

\section{REFERENCIAS}

1. Globocan 2002. International Agency for research on Cancer. Acceso Junio de 2007. Disponible en www.iarc.fr

2. Parkin DM, Whelan SL, Ferlay J, Teppo L and Thomas DB (eds) (2003). Cancer incidence in five continents, Vol VIII, IARC Scientific Publications No. 155, Lyon, IARC.
3. Jemal A, Siegel R, Ward E, Murria T, Xu J, Thun MJ. Cancer statistics, 2007. CA Cancer J Clin 2007;57(1):43-66.

4. Ferlay J, Bray F, Pisani P, Parkin DM (2001) GLOBOCAN 2000: Cancer Incidence, Mortality and Prevalence Worldwide, Version 1.0. IARC Cancer Base No 5. Lyon, IARCPress.

5. Stein JP, Lieskovsky G, Cote R, Groshen S, Feng AC, Boyd S, et al. Radical cystectomy in the treatment of invasive bladder cancer: long-term results in 1.054 patients. J Clin Oncol 2001;19(3):666-675.

6. Sternberg CN, Donat M, Bellmut J, Millikan RE, Stadler W, De Mulder P, et al. Chemotherapy for bladder cancer: Treatment guidelines for neoadjuvant chemotherapy, bladder preservation, adjuvant chemotherapy, and metastatic cancer. Urology 2007;69 (Supp 1A):62-79.

7. Soloway MS, López AE, Patel J, Lu Y. Results of radical cystectomy for transitional cell carcinoma of the bladder and the effects of chemotherapy. Cancer 1994;73(7):1926-1931.

8. Frazier HA, Robertson JE, Dodge RK, Paulson DF. The value of pathologic factors in predicting cancer-specific survival among patients treated with radical cystectomy for transitional cell carcinoma of the bladder and prostate. Cancer 1993;71(12): 3993-4001.

9. Nishiyama H, Habuchi T, Watanabe J, Teramukai S, Tada $H$, Ono Y, et al. Clinical outcome of a large-scale multi-institutional retrospective study for locally advanced bladder cancer: a survey including 1131 patients treated during 1990-2000 in Japan. Eur Urol 2004;45(2):176-181.

10. Herr HW. Extent of surgery and pathology evaluation has an impact on bladder cancer outcome after radical cystectomy. Urology 2003;61(1):105-108.

11. Thieblemont C, Fendler JP, Trillet-Lenoir V, Petris C, Chauvin F, Brunat-Mentigny M, et al. Prognostic factors of survival in infiltrating urothelial bladder carcinoma. A retrospective study of 158 patients treated by radical cystectomy. Bull cancer 1996;83(2):139-146.

12. Takahashi A, Tsukamoto T, Tobisu K, Shinohara N, Sato K, Tomita Y, et al. Radical cystectomy for invasive bladder cancer: results of a multi-institutional pooled analysis. Jpn J Clin Oncol 2004;34(1): 14-19.

13. Cheng L, Weaver AL, Leibivich BC, Ramnani DM, Neumann RX, Sherer BG, et al. Predicting the survival of bladder carcinoma in patients treated with radical cystectomy. Cancer 2000; 88(10):2326-2332.

14. Shariat SF, Karakiewicz PI, Palapattu GS, Lotan Y, Rogers CG, Amiel GE, et al. Outcomes of radical cystectomy for transitional cell carcinoma of the bladder: a contemporary series from the bladder cancer research consortium. J Urol 2006;176(6 Pt1):2414-2422.

15. Bassi P, Ferrante GD, Piazza N, Spinadin R, Carando R, Pappagallo G, et al. Prognostic factors of outcome alter radical cystectomy for bladder cancer: a retrospective study of a homogeneous patient cohort. J Urol 1999; 161(5):1494-1497.

16. Hautmann RE, Gschwend JE, de Petriconi R, Kron M, Volkmer B. Cystectomy for transitional cell carcinoma of the bladder: results of a surgery only series in the neobladder era. J Urol 2006;176(2):486-492.

17. Hara S, Miyake H, Fujisawa M, Okada H, Arakawa S, Kamidono S, et al. Prognostic variables in patients who have undergone radical cystectomy for transitional cell carcinoma of the bladder. Jpn J Clin Oncol 2001;31(8):399-402.

18. Antunes AA, Nesrallah LJ, Dal Óglio M, Ferreira YA, Passerotti $\mathrm{CC}$, Leite KR, et al. Analysis of prognosis factors in patients with transitional cell carcinoma of the bladder treated with radical cystectomy. Int Braz J Urol 2006;32(1):35-42. 
19. Segura Martín M, Salinas Sánchez AS, Lorenzo Romero JG, Hernández Millán I, Jiménez Bachs JM, Virseda Rodríguez JA. Supervivencia en pacientes sometidos a cistectomía radical por carcinoma vesical. Actas Urol Esp 2001;25(10):737745.

20. Dalbagni G, Genera E, Hashibe M, Zhang Z, Russo P, Herr H, et al. Cystectomy for bladder cancer: a contemporary series. J Urol 2001;165(4): 1111-1116.

21. Harada K, Sakai I, Kurahashi T, Muramaki M, Yamanaka K, Hara I, Eto H, Miyake H. Clinicopatological features of recurrence after radical cystectomy for patients with transitional cell carcinoma of the bladder. Int Urol Nephron 2006;38(1):4955.

22. Louis-Johnsun MW, Braslis KG, Murphy DL, Neerhut GJ, Grills R. Radical cystectomy for primary bladder cancer: a 10 year review. ANZ J Sur 2007; 77(4):265-269.

23. Millar JL, Frydenberg M, Toner G, Syme R, Thursfield V, Giles GG. Management of muscle-invasive bladder cancer in Victoria, 1990-1995. ANZ J Surg 2006;76(3):113-119.

24. Solsona E, Iborra I, Dumont R, Rubio J, Casanova Lalmenar S. Risk groups in patients with bladder cancer treated with radical cystectomy: statical and clinical model improving homogeneity. J Urol 2005;174(4 Pt 1):1226-1230.

25. Studer UE, Bacchi M, Biedermann C, Jaeger P, Kraft R, Mazzucchelli L, et al. Adjuvant cisplatin chemotherapy following cystectomy for bladder cancer: results of a prospective randomized trial. J Urol 1994;152(1):81-84.
26. Skinner DG, Daniels JR, Russell CA, Lieskovsky G, Boyd SD, Nichols $\mathrm{P}$, et al. The role of adjuvant chemotherapy following cystectomy for invasive bladder cancer: a prospective comparative trial. J Urol. 1991;145(3):459-464.

27. Stöckle M, Meyenburg W, Wellek S, Voges G, Gertenbach U, Thüroff JW, et al. Advanced bladder cancer (stages pT3b, pT4a, pN1 and pN2): improved survival after radical cystectomy and 3 adjuvant cycles of chemotherapy. Results of a controlled prospective study. J Urol 1992;148(2 Pt 1):302-306.

28. Freiha F, Reese J, Torti FM. A randomized trial of radical cystectomy versus radical cystectomy plus cisplatin, vinblastine and methotrexate chemotherapy for muscle invasive bladder cancer. J Urol 1996;155(2):495-499.

29. Sylvester R, Stenberg C. The role of adjuvant combination chemotherapy after cystectomy in locally advanced bladder cancer: What we do not know and why. Ann Oncol 2000;11(7):851-856.

30. Guidelines on bladder cancer: muscle-invasive and metastatic. European Urology Association of Urology 2006. Disponible en http://www.uroweb.org/nc/professional-resources /guidelines/online/

Correspondencia autor: Dr. Juan I. Monzó Garnier Servicio Urología. Fundación Jiménez Díaz

Avda. Reyes Católicos 2 - 28040 Madrid. Tel.: 915504800

E-mail autor: juanimonzo@hotmail.com

Información artículo: Original - Cáncer de vejiga

Trabajo recibido: mayo 2008

Trabajo aceptado: diciembre 2008 